\title{
REVERSE CURRENT IN SOLAR FLARES
}

by

J.W. Knight and P.A. Sturrock

$($ SASA-CB-1494C4) BEVEBSE CUBEANI IN SOLAB

FLAEZS (StanfCrd Univ.) 23 f HC $B 02 / \mathrm{MF} \mathrm{BO} 1$

CSCI O $3 \mathrm{E}$

1177-16978

UnCius

$63 / 9213305$

National Aeronautics and Space Administration

Grant NGL 05-020-272

Office of Naval Research

Contract N00014-75-C-0673

Heproduction in whole or in part

is permitted for any purpose of

the United States Government.

SUIPR Report No. 678

December 1976

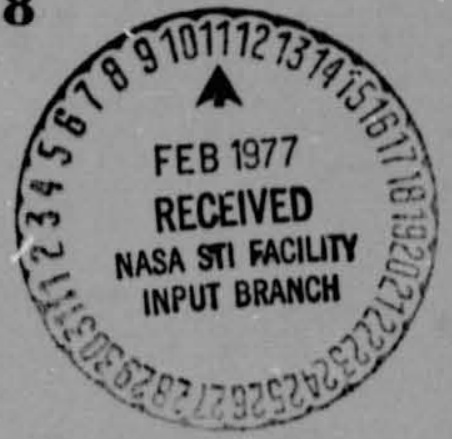

INSTITUTE FOR PLASMA RESEARCH STANFORD UNIVERSITY, STANFORD, CALIFORNIA 


\section{REVERSE CURRENT IN SOLAR FLARES}

by

J.W. Knight ${ }^{*}$ and P.A. Sturrock ${ }^{*}$

National Aeronautics and Space Administration

Grant NGL 05-020-272

Office of Naval Research

Contract No0014-75-C-0673

SUIPR Report No. 678

December 1976

$$
\begin{gathered}
\text { Institute for Plasma Research } \\
\text { Stanford University } \\
\text { Stanford, California }
\end{gathered}
$$

\footnotetext{
XA1so Department of Applied Physics
} 
REVERSE CURRENT IN SOLAR FLARES

by

J.W. Knight and P.A. Sturrock Institute for Plasma Research and

Department of Applied Physics

Stanford University

Stanford, California

\begin{abstract}
We examine the proposal that impulsive $x$-ray bursts are produced by high-energy electrons streaming from the corona to the chromosphere. We find that the currents associated with these streams are so high that either the streams do not exist or their current is neutralized by a reverse current.

Analysis of a simple model indicates that the primary electron stream leads to the development of an electric field in the ambient corona which (a) decelerates the primary beam and (b) produces a neutralizing reverse current. It appears that, in some circumstances, this electric field could prevent the primary beam from reaching the chromosphere. In any case, the electric field acts as an energy exchange mechanism; extracting kinetic energy from the primary beam and using it to heat the ambient plasma. This heating is typically so rapid that it must be expected to have important dynamical consequences.
\end{abstract}




\section{Introduction}

Nuch of the line emission from solar flares must originate in plasma of chromospheric density. On the other hand, the corona offers a much larger volume for the storage of magnetic energy than does the chromosphere. This has led to models in which free magnetic energy, released in the corona, propagates to the chromosphere in the form of streams of high-energy particles (Sweet, 1969; sturrock, 1974). Observational evidence for high-energy electron streams comes from analysis of microwave bursts and Impulstive $x$-ray bursts (Kane, 1972). Evidence concerning the origin 7 impulsive $x$-ray bursts is conflicting and it is possible that some radiation originates at the chromosphere, as analyzed by Brown (1972), Hudson (1972) and Petrosian (1973), and that some originates in the corona as proposed by de Jager and Kundu (1963), Kane and Anderson (1970) and Kane (1972).

It has recently occurred to us, and to other solar physicists including Brown (1976) and Colgate (1976), that the electrical currents associated with the proposed electron streams are so high that the streams either do not exist or are neutralized by reverse currents in the ambient plasma. The reason for this statement are discussed briefly in Section II. An idealized model in which the primary current is neutralized by a reverse current is presented in Section III and applied to solar flares in Section IV. Some of the consequences of these considerations are discussed in Section $V$. 


\section{Magnetic Field Associated with Proposed Electron Stream}

If energy released in the corona propagates to the chromosphere in the form of high-energy electrons, this electron stream will constitute an electrical current. If $\mathrm{N}$ is the total number of electrons streaming downward over the duration $\tau$ (soc.) of the impulsive phase, the magnitude $\mathrm{J}$ (e.m.u.) of the current may be estimated from

$$
J \sim e c^{-1} N T^{-1}
$$

If the transverse and Iongitudinal dimensions of the stream are of order $\mathrm{L}(\mathrm{cm}$.$) , an estimate of the strength B$ (gauss) of the magnetic fleld produced by the stream is given by

$$
\mathrm{B} \sim 2 \mathrm{~J} \mathrm{~L}^{-1}
$$

and the total energy $U$ (erg) of this magnetic field may be estimated from

$$
\mathrm{U} \sim \frac{1}{8 \pi} \mathrm{L}^{3} \mathrm{~B}^{2} \sim \frac{1}{2 \pi} \mathrm{J}^{2} \mathrm{~L}
$$

which becomes

$$
\mathrm{U} \sim \frac{1}{2 \pi} \mathrm{e}^{2} \mathrm{c}^{-2} \mathrm{~N}^{2} \tau^{-2} \mathrm{~L} \sim 10^{-40.4} \mathrm{~N}^{2} \mathrm{~T}^{-2} \mathrm{~L} .
$$

Kane and Anderson (1970) estimate the total energy involved in a typical small flare to be $\sim 10^{29} \mathrm{erg}$, the time scale to be $\sim 10^{2} \mathrm{sec}$, the characteristic length scale to be $\sim 10^{8.5} \mathrm{~cm}$, and infer from the $x$-ray data that the total number of energetic electrons 1 s $\sim 10^{35}$. For these values, the above formulas lead to estimates $\mathrm{J} \sim 10^{13.2}, \mathrm{~B} \sim 10^{5}$ and $U \sim 10^{34}$. It is clear that this model, involving energy transport by 
an unileutralized electron beam, leads to unacceptably high values of the magnetic field of the beam and magnetic energy associated with the beam. When one considers large flares rather than small flares, one faces the same dfficulty.

One resolution of this difficulty is to abandon the assumption that energy is transported from the corona to the chromosphere by an electron stream. One might assume, for instance, that energy ts transported as heat flux. The above parameters for a small flare then indicate that the mean heat flux $\mathrm{F}$ (erg $\mathrm{cm}^{-2} \mathrm{~s}^{-1}$ ) would be $\sim 10^{10}$. From the formula approprfate for a fully ionized plasma (Spitzer, 1962),

$$
\mathrm{F}=x \frac{\mathrm{d} \mathrm{T}}{\mathrm{dz}} \sim 10^{-6} \mathrm{~T}^{5 / 2} \frac{\mathrm{d} \mathrm{T}}{\mathrm{dz}}
$$

we find that the electron temperature $\mathrm{T}\left({ }^{\circ} \mathrm{K}\right)$ must be at least $10^{7}$ to give the required heat flux. For the concept of heat flux to be valid, the mean free path must be small compared with the length scale for temperature variation, e.g. $10^{-1} \mathrm{~L}$, which is $\sim 10^{7.5}$ for the case now being considered. Since the mean free path $\gamma$ for electron-electron and electron-ion collisions is given by

$$
Y \approx 10^{4} \mathrm{r}^{2} \mathrm{n}^{-1}
$$

we see that the electron density $n\left(\mathrm{~cm}^{-3}\right)$ must be at least $10^{10.5}$. This is substantially higher than is typical in the normal corona at the initiation of a flare, although densities of this magnitude do build up in the course of a flare. This indicates that, although heat conduction may be an important, and perhaps the dominant, mechanism of energy transfer in the later stages of a flare, it cannot be the principal 
mode of energy transfer at flare onset. (One should, however, bear in mind the possibility that instabilities may lead to modification of the transport properties of the plasma, modifying both the heat conduction and effective mean free path.) 


\section{Model of Electron Stream and Reverse Current}

It is well known that a plasma tends to preserve charge neutrality. A process which tends to give an excess positive or negative charge in some region will lead to electric fields acting upon the plasma. Movement. of electrons in response to this electric fleld wi1? then restore charge neutrality. One expects that analogous processe wi.11 also tend to malntain current neutrality. If an electron beam is suddenly fntroduced into a plasma, there is a sudden change in the magnetic field structure which will develop induced electric fields opposing the primary current. For this reason, we should investigate the possfbility that a reverse current develops in the corona balancing (or almost balancing) the primary current due to an assumed electron stream flowing from the corona to the chromosphere.

Our constderations are substantialiy simplified if we consider a symmetrical flux tube rooted in the photosphere and extending up into the corona, and assume that electrons are accelerated at the top of the flux tube by the development of stochastic electric fields or by some other mechanism. The ejection of these electrons down towards the chromosphere then leads to a charge imbalance which leads to the development of an electric field of such a magnitude that charge balance will be restored. If there is no net change in charge density, whatever current flows along the flux tube must be constant along the length of the tube. If the tube is symmetrical, this current must be zero. Hence the strong tendency of plasma to remain charge neutral implies that any primary current will generate a neutralizing secondary reverse current. 
In order to simplify this exploratory analysis, we consider only a steady-state situation. We also assume that the background plasma can be adequately described by a Maxwellian velocity distribution and use transport coefficients based on this assumption (Spitzer, 1962). We are interested in the case that the primary electron stream is composed of high-energy electrons which consequently have long mean Eree paths. However, we sha1l find that an electric field develops which decelerates the electron stream. But when the electron energy becomes comparable with the thermal energy, the mean free path w111 be sufficiently short that the primary electrons will merge with the background plasma. As a simple representation of this process, we ignore collisions in discussing the primary beam but we assume that an electron of the primary beam is absorbed into the background plasma when it is decelerated to zero energy.

If, as a further simplification, we consider a magnetic flux tube of constant cross section, we may use the following simple one-dimensional form of the viasov equation:

$$
\frac{v f f}{\partial s}+\frac{e}{m} \frac{d p}{d s} \frac{\partial f}{\partial v}=0
$$

where $s$ measures arc length along the tube, $v$ is velocity (along the tube), $f(s, v)$ is the velocity distribution function of the primary electron stream, and $\varphi$ is the electrostatic potential.

At the top of the loop $(s=0)$, the primary electron stream is moving with positive velocity on the side corresponding to increasing s. Hence we may, without ambiguity, express $f$ in terms of $\psi$ defined by

$$
\psi=\frac{m v^{2}}{2 e} .
$$


The inftal distribution function may therefore be expressed as

$$
E(0, v)=F(\psi)
$$

With this initial condition, we find that the solution of the vlasov equation (3.1) 1/s

$$
f(s, v)=F(\downarrow-\varphi)
$$

The current density $j_{s}$ (e.m.u.) in the primary electron stream is given by

$$
j_{s}=-\frac{e}{c} \int_{0}^{\infty} E(s, v) v d v
$$

which may be expressed as

$$
j_{s}=-\frac{e^{2}}{m c} \int_{0}^{\infty} F(\psi-\varphi) d \psi .
$$

Since $\varphi$ wi11 prove to be negative in the region of interest, it is convenient to write

$$
\theta=-\varphi
$$

so that (3.6) may be reexpressed as

$$
f_{s}=-\frac{e^{2}}{m c} \int_{\theta}^{\infty} F(x) d x
$$

Charged neutrality requires that

$$
j_{p}+j_{s}=0
$$

where $j_{p}$ is the secondary current induced in the background plasma. We here assume that the density and temperature are such that this may be 
represented by Ohm's law,

$$
J_{p}=\sigma E=\sigma \frac{d \theta}{d s}
$$

where $\sigma$ is the elactrical conductivity expressed in modffind gaussian units.

It is convenient to introduce a new independent valrable $\xi$ to replace $s$, by the relationship

$$
\mathrm{d} \xi=\frac{\mathrm{ds}}{\sigma(\mathrm{s})}
$$

Then, on substituting equations $(3.8)$ and $(3.10)$ into $(3.9)$ and differentiating with respect to 5 , we obtain

$$
\frac{d^{2} \theta}{d \xi^{2}}+\frac{e^{2}}{m c} F(\theta) \frac{d \theta}{d \xi}=0
$$

It is convenient to solve this equation for 5 in terms of $\theta$,

$$
\xi=x(\theta),
$$

rather than vice versa. Equation (3.13) becomes

$$
\frac{m c}{e^{2}}\left(\frac{d X}{d \theta}\right)^{-2} \frac{d^{2} x}{d \theta^{2}}=r(\theta)
$$

which may be integrated once to give

$$
\frac{m c}{2}\left[\left(\frac{d \theta}{d \xi}\right)_{\xi=0}-\left(\frac{d x}{d \theta}\right)^{-1}\right]=\int_{0}^{\theta} F(\dot{\theta}) \cdot d \theta,
$$

if we assume that $\theta=0(\varphi=0)$ and $x=0(\xi=0)$ at $s=0$. We ind, from $(3.9),(3.9)$ and $(3.10)$, that 


$$
\frac{e^{?}}{m c}\left(\frac{d \theta}{d \xi}\right)_{\xi=0}=\int_{0}^{\infty} F(\hat{\theta}) d \theta^{-1} .
$$

Hence equation (3.15) becomes

$$
\frac{d X}{d \theta}=\frac{m c}{e^{2}}\left[\int_{0}^{\infty} F(\theta) d \theta\right]^{-1} .
$$




\section{Specific Mode1}

It ts now convenient to Introduce a spectefc form for $F(\psi)$,

$$
F(\psi)=k\left(\psi_{0}+\psi\right)^{-\gamma}
$$

This is a power-law distribution at high energy which flattens at low energy, the "knee" being characterized by $\varphi_{0}$.

We introduce the symbol $\mathrm{H}(\psi, \mathrm{s})$ for the flux df electrons $\left(\mathrm{cm}^{-?} \mathrm{~s}^{-1}\right)$ of energy exceeding el at the postition $s$

$$
H(\psi, s)=\frac{e}{m} \int_{\psi}^{\infty} F(\dot{\psi}+\theta(s)) d \dot{\psi} .
$$

If the initial flux is written as $H_{0}(\psi)$, we find that

$$
\mathrm{H}_{0}(\psi)=\frac{\mathrm{ek}}{(\gamma-1} ;-\left(\psi_{0}+\psi\right)^{-\gamma+1},
$$

5: that the total particle flux is given by

$$
H_{0}(0)=\frac{e K}{(\gamma-1) m} \psi_{0}^{-\gamma+1}
$$

With the form (4.1) for $F(\psi)$, equation (3.17) integrates to ive

$$
x(\theta)=\frac{\gamma-1}{\gamma} \frac{m c}{e^{2} K}\left[\left(\psi_{0}+\theta\right)^{\gamma}-\psi_{0}^{\gamma}\right] \text {. }
$$

We easily obtaln from (4.5) an expression for the (neyative) electric potential $\theta$ in terms of the modified distance measure 5 :

$$
\theta(\xi)=\left[\psi_{0}^{Y}+\frac{\gamma}{\gamma-1} \frac{e^{2} K}{m c} \varepsilon\right]^{1 / \gamma}-\psi_{0} .
$$

Hence, from ( 4.2$)$, we find that

$$
\left.H(\psi, s)=\frac{e K}{(\gamma+1) m}\left[\psi \cdot \psi_{0}^{\gamma}+\frac{\gamma}{\gamma-1} \frac{e^{2} K}{m c} \xi\right\}^{1 / \gamma}\right]^{-\frac{(\gamma-1)}{\gamma}}
$$




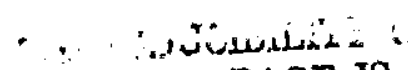

2.UISINAL PAGE IS P:

On noting that the electric current carried by the stream is related to $H(\psi, s)$ by

$$
j_{s}(s)=-\frac{e}{c} H(0, s),
$$

we see that

$$
J_{s}(s):-\frac{e^{2} K}{(\gamma-1) m c}\left[\psi_{0}^{\gamma}+\frac{\gamma}{\gamma-1} \frac{e^{2} K}{m c}\right]^{-\frac{\gamma-1}{\gamma}}
$$

In order to specify the current, particle flux and electric field as functions of $\mathrm{s}$, we must adopt a specific form for $\sigma(\mathrm{s})$. A conventent approximation to the structure of the solar atmosphere, which is expressible in analytic form, is provided by the constant heat flux model. If we now assume that $s$ measures distance vertically downwards from the corona, and that $\mathrm{n}=\mathrm{n}_{0}, \mathrm{~T}=\mathrm{T}_{0}$, at $\mathrm{s}=0$, this model (Adams and Sturrock, 1975) yields the following expressions:-

$$
\begin{gathered}
\mathrm{T}(\mathrm{s})=\left(\mathrm{T}_{0}^{7 / 2} \cdot \mathrm{bFs}\right)^{2 / 7} \\
\mathrm{n}(\mathrm{s})=\mathrm{n}_{0}\left(\mathrm{~T}_{0} / \mathrm{T}(\mathrm{s})\right) \exp \left\{-\frac{\mathrm{a}}{\mathrm{F}}\left[\left(\mathrm{T}_{0}^{7 / 2}-\mathrm{bFs}\right)^{5 / 7}-\mathrm{T}_{0}^{5 / 2}\right]\right\},
\end{gathered}
$$

where $a \approx 10^{-10.21}, \mathrm{~b} \approx 10^{6.58}$, and $\mathrm{F}$ (erg $\mathrm{cm}^{-2} \mathrm{~s}^{-1}$ ) is the downward heat flux.

The electrical conductivity, in modffied gaussian units, may be derived from the expression given by Spitzer (1962):

$$
\sigma=g \mathrm{~T}^{3 / 8}
$$

where $g \approx 10^{-3.64}$. Hence we find, from $(3.11)$, that $\xi$ is related to 
s by

$$
\xi=\frac{7}{4} \frac{1}{\mathrm{bgF}}\left[\mathrm{T}_{0}^{2}-\left(\mathrm{r}_{0}^{\mathrm{r} / 2}-\mathrm{bFs}\right)^{4 / 7}\right]
$$

Our model is then completely spectified by the choice of the coronal temperature, the coronal density, the coronal heat $f l u x, \gamma$, the energy corresponding to $\Psi_{0}$, and the injected energetic electron flux. For the coronal parameters, we have adopted values typical of the corona above an active region (Noyes, 1971):

$$
\begin{aligned}
& \mathrm{T}=3 \times 10^{6} \mathrm{~K} \\
& \mathrm{n}=10^{9} \mathrm{~cm}^{-3} \\
& F=5 \times 10^{6} \mathrm{erg} \mathrm{cm}^{-2} \mathrm{sec}^{-1} \text {. }
\end{aligned}
$$

We choose $\psi_{O}$ to correspond to $25 \mathrm{kev}$ and we choose $\gamma=2.5$, corresponding to an siray spectral index of $\sim 3-3.5$ (Brown, 1975), which is a reasonable vatu for the spectral index at peak emission (Datlowe, 1975). The fraction of tio beam energy deposited, and the total ine:-gr deposited by joule heating witween $\mathrm{T}=3 \times 10^{6} \mathrm{~K}$ and $\mathrm{T}=3 \times 10^{4} \mathrm{~K}$ as . a function of the energetic electson flux, are displayed in Figure 1. For a flare area of $10^{19.5} \mathrm{~cm}^{2}$, the tinergetic electron flux inferred from a large impulsive $x$-ray burst correswonds to $\sim 10^{17} \mathrm{~cm}^{-2} \mathrm{sec}^{-1}$ (Hoyng et al., 1975). Figure 2 illustrates :he energy deposition rate due to joule heating as a function of tempirature of the atmosphere for this injected energetic electron flux. The orlinate of Figure 2 is the time required to raise the ambient plasma temporature by $10^{7} \mathrm{~K}$. 


\section{Discussion}

For the adopted mode1, Figure 2 shows that the steady-state assumption is not justified for the energetic electron flux required to produce the observed $x$-ray flux by thick target bremsstrahlung. The atmosphere, especially the dense low-temperature region, will be heated rapidly to a high temperature. Once the plasma is raised to a few times $10^{7} \mathrm{~K}$, ohmic losses will become unimportant since the joule heating rate is inversely proportional to the electrical conductivity. Joule heating is a more effective mechanism for heating the plasma than collisional losses from the energetic electrons, because the ohmic losses are causad by thermal electrons in the reverse curretit which have much shorter mean free paths than the energetic electrons. It is likely that a larger fraction of the beam energy is deposited than is indicated in Figure 1 for two reasons. First, the constant heat flux model underestimates the extent of the low temperature region where the ohmic losses are largest. Second, if the drift velocity associated with the reverse current becomes too large, instabilities can develop and reduce the electrical conductivity of the plasma. If the conductivity of the plasma is low enough, the electric field that develops may be large enough to effectively prevent the stream from reaching the chromosphere.

The time scale for heating the dense low-temperature plasma is comparable with the time scales characteristic of impulsive x-ray bursts (Hoyng et al., 1975). It is possible that thermal bremsstrahlung from the rapidly heated plasma can account for a significant portion of the observed impulsive $x$-ray flux. Hence this mechanism offers an explanation of the fact that some flares first produce high-energy $x$-ray emission 
near the top of a loop rather than at the foot-points of the loop (Brueckner, 1976). Another important consequence of this process is that, if thermal emission can account for a substantial fraction of the impulsive flux up to $\sim 50 \mathrm{kev}$, then the number of electrons required to produce the non-thermal $x$-ray flux is greatly reduced (Brown, 1975).

The rapid heating of the plasma will also cause a large pressure imbalance. The time $T$ (sec.) for the plasma to respond to pressure changes by bulk motions can be estimated from

$$
\tau \sim \frac{\mathrm{L}}{\mathrm{V}_{\mathrm{S}}},
$$

where $L$ is a characteristic length and $V_{s}$ is the fon thermal velocity. Even for a temperature as large as $10^{7} \mathrm{~K}$, this time is long $\left(10^{2}\right.$ sec) compared to the heating time for a length scale of $10^{9.7} \mathrm{~cm}$, so that the plasma density wili not change appreciably during the heating. In the constant heat flux model we have adopted, the density at $3 \times 10^{4} \mathrm{~K}$ is approximately 100 times the density at $3 \times 10^{6} \mathrm{~K}$. If both these regions are heated to a temperature of a few times $10^{7} \mathrm{~K}$, the resulting pressure imbalance must be expected to produce explosive evaporation of high density plasma into the corona.

A more realistic time-dependent model is necessary to account for the complicated phenomena observed in solar flares. It is also possible that collective effects are important and that the assumption of a Maxwellian velocity distribution for the ambient plasma must be abandoned. However, our analysis of reverse current in terms of an idealized model strongly suggests that joule heating is likely to play an important role in the heating of solar plasma during a flare. 


\section{Acknowledgements}

This work was supported by the National Aeronautics and Space Administration under grant NGL $05-020-272$ and the Tffice of Naval Research under contract No0014-75-C-0673. 


\section{References}

Brown, J.C. 1972, Solar Phys. 26, 441.

Brown, J.C. 1975, Solar Gamma-, X- and EUV Radiation (S.R. Kane, ed.)

(Dordrecht: Reide1) 245.

Brown, J.C. 1976, Presentation to Commission 10, I.A.U. General Assembly, (Grenoble, France, August 1976).

Brueckner, G.E. 1976, Phi1. Trans. Roy. Soc. (A) 281, 443.

Colgate, S.A. 1976, "Phenomenological Model of Solar Flares", to be published.

Datlowe, D.W. 1975, Solar Gamma-, X- and EUV Radiation (S.R. Kane, ed.)

(Dordrecht: Reide1) 191.

de Jager, C. and Kundu, M.R. 1963, Space Research 3, 836.

Hoyng, P., Brown, J.C., Stevens, G. and van Beek, H.F. 1975, Solar Gamma-

X- and EUV Radiation (S.R. Kane, ed.) (Dordrecht: Reidel) 233.

Hudson, H.S. 1972, Solar Phys. 24, 414.

Kane, S.R. 1972, Proc. Symposium on High Energy Phenomena on the Sun

(R. Ramaty and R.G. Stone, eds.), NASA SP-342, 55 .

Kane, S.R. and Anderson, R.A. 1970, Ap. J. 162, 1003.

Noyes, R.W. 1971, Ann. Rev. Astron. Ap. 2, 209.

Petrosian, V. 1973, Ap. J. $186,291$.

Peterson, C.E. and Winckler, J.R. 1959, J.G.R. 64, 697.

Sturrock, P.A. 1974, Corona1 Disturbances (G. Newkirk, ed.) (Dordrecht:

Reide1), 437 .

Sweet, P.A. 1969, Ann. Rev. Astron. Ap. Z, 149. 


\section{Figure Captions}

. Figure 1. The fraction of the beam energy deposited (solid curve! and the total energy deposited (broken curve) by joule heating as a function of the energetic particle number flux.

Figure 2. Time $\tau_{H}$ required for ohmic losses to heat the plasma by $10^{7} \mathrm{~K}$ as a function of the ambient plasma temperature. 


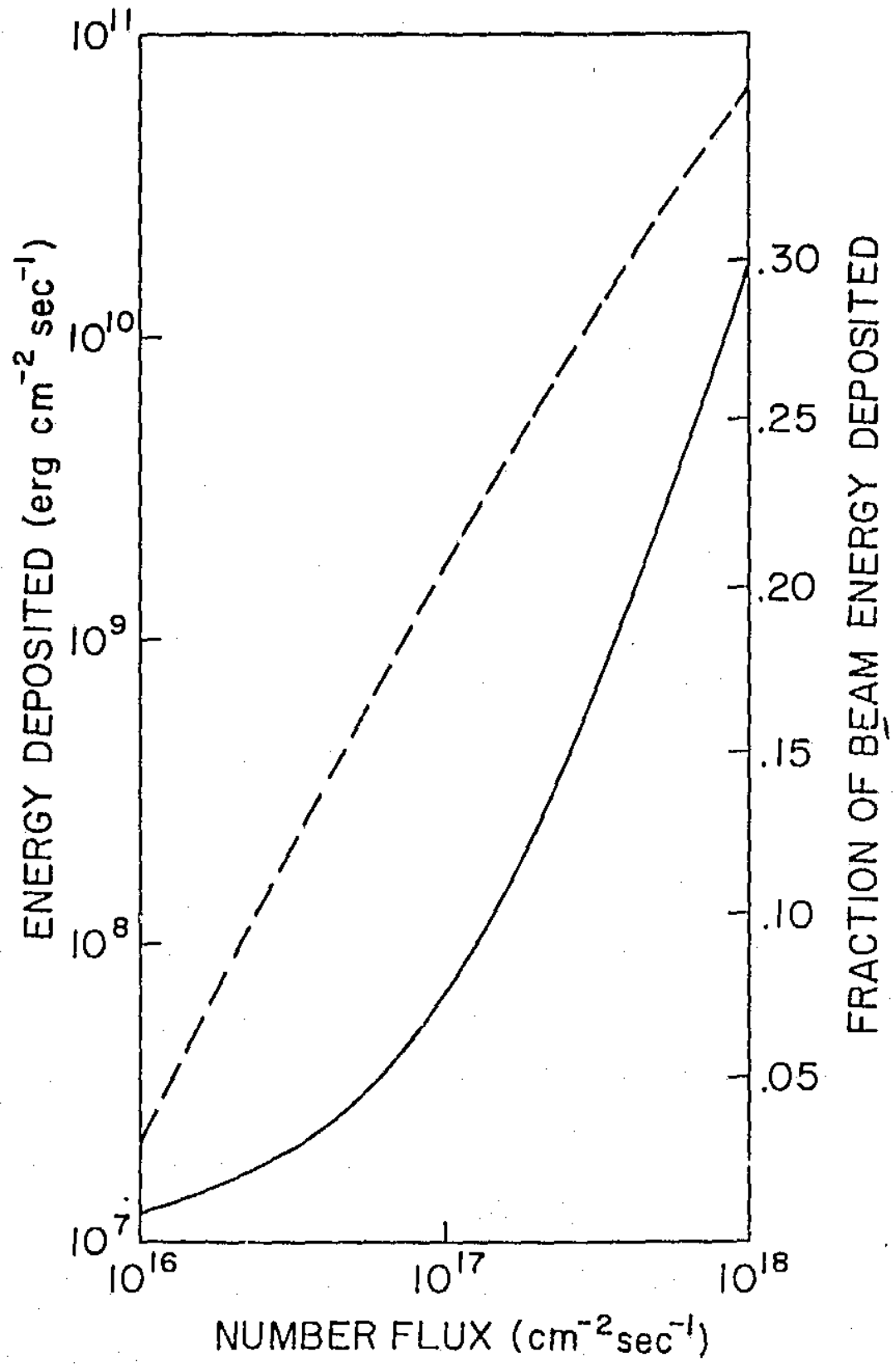

Figure 1 


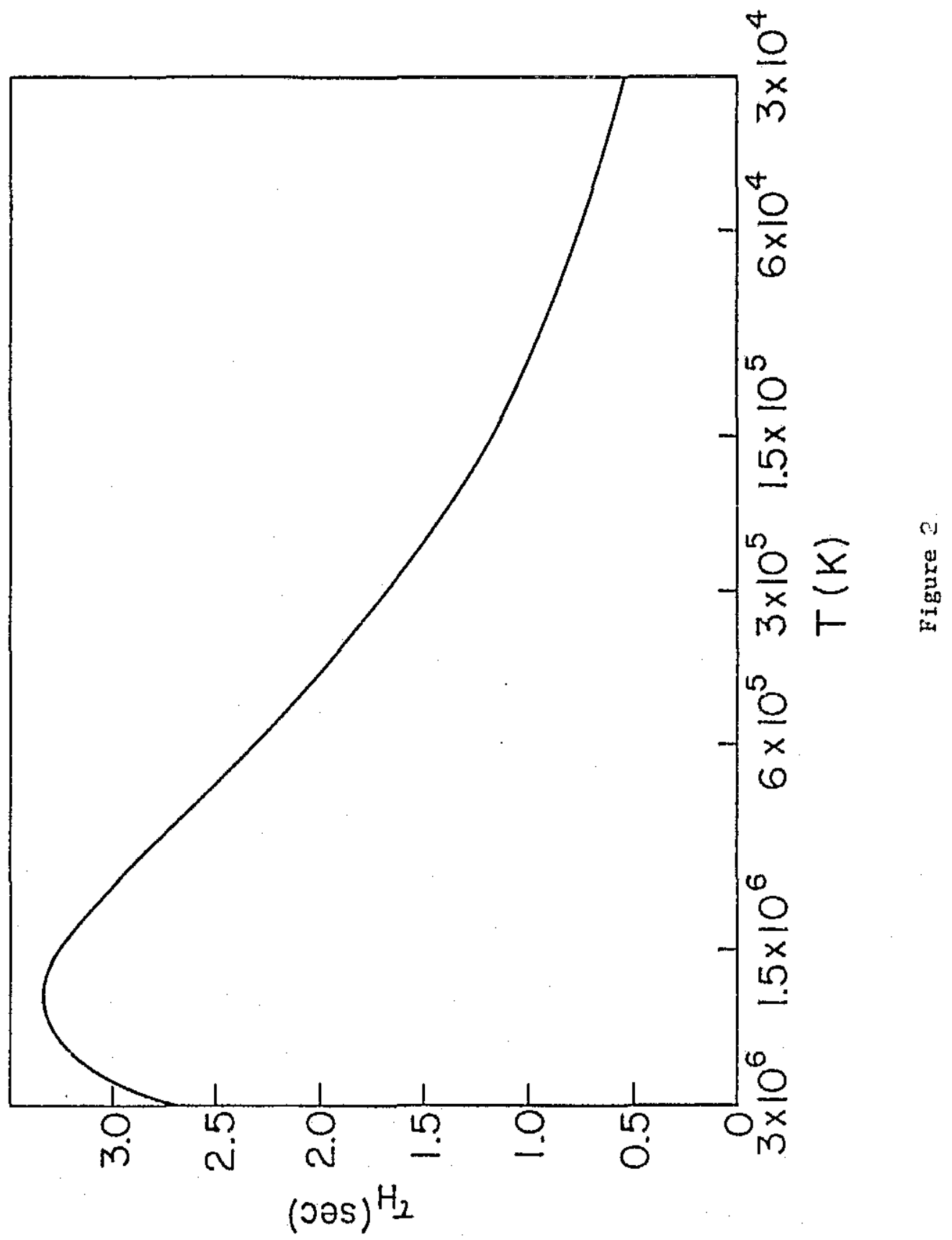

